\title{
Hospital Admission Less than 30 Days after Chemotherapy: Results from a Chemotherapy-Specific Morbidity and Mortality Conference in Gynecologic Oncology
}

\author{
Lauren Philp1, Tracilyn Hall ${ }^{2}$, Lisa Diver ${ }^{3}$, Annekathryn Goodman ${ }^{1}$ \\ ${ }^{1}$ Department of Obstetrics and Gynecology, Division of Gynecologic Oncology, Massachusetts General Hospital, Boston, MA, USA \\ ${ }^{2}$ Department of Obstetrics and Gynecology, Division of Gynecologic Oncology, Baylor College of Medicine, Houston, TX, USA \\ ${ }^{3}$ Department of Obstetrics and Gynecology, Division of Gynecologic Oncology, Stanford University, Palo Alto, CA, USA \\ Email:lphilp@mgh.harvard.edu
}

How to cite this paper: Philp, L., Hall, T., Diver, L. and Goodman, A. (2020) Hospital Admission Less than 30 Days after Chemotherapy: Results from a Chemotherapy-Specific Morbidity and Mortality Conference in Gynecologic Oncology. Journal of Cancer Therapy, 11, 377-387.

https://doi.org/10.4236/jct.2020.116033

Received: June 2, 2020

Accepted: June 25, 2020

Published: June 28, 2020

Copyright $\odot 2020$ by author(s) and Scientific Research Publishing Inc. This work is licensed under the Creative Commons Attribution International License (CC BY 4.0).

http://creativecommons.org/licenses/by/4.0/

(c) (i) Open Access

\begin{abstract}
Introduction: Morbidity and Mortality $(M \& M)$ rounds can identify adverse events and improve patient safety however adoption in cancer centers is not routine. Herein we report the results of a chemotherapy-specific gynecologic oncology M\&M rounds and identify reasons for hospital admission < 30 days after chemotherapy treatment. Methods: Between July 2014 and April 2016, all admissions $<30$ days from chemotherapy administration were prospectively collected along with clinical data. Admissions were described and classified as planned or unplanned and as associated with chemotherapy or with underlying disease. Results: 585 patients were admitted, $78 \%$ of whom had ovarian cancer and $43 \%$ of whom had recurrent disease. Overall, $47 \%$ of admissions were unplanned and these were significantly longer than planned admissions ( 5.6 vs. 2.4 days, $\mathrm{p}=0.0003$ ). Of unplanned admissions, $43 \%$ were due to chemotherapy, and $57 \%$ were due to disease burden. $74 \%$ of patients had received $>1$ prior line of chemotherapy, and $22 \%$ were on clinical trial. The most common causes of unplanned admission were nausea, vomiting or failure to thrive (28.9\%), fever (17.9\%) and small bowel obstruction (19.9\%). Conclusions: There is a high rate of unplanned admission $<30$ days after chemotherapy and patients with ovarian cancer and recurrent disease are at the highest risk. This information can be used to counsel patients about complications of chemotherapy and to improve supportive management. M\&M conferences surrounding unplanned admissions after chemotherapy may help guide therapy, encourage best supportive care, and prompt re-evaluation of treatment goals in heavily pretreated patients with recurrent.
\end{abstract}




\section{Keywords}

Gynecologic Oncology, Quality Improvement, Chemotherapy

\section{Introduction}

Morbidity and mortality conferences (M\&M) have been incorporated into clinical practice as a way to discuss adverse events and errors associated with medical treatment [1]. These non-punitive conferences involve the analysis of complications using peer review in an attempt to identify systems-based approaches to improve patient care. These forums have been shown to promote disclosure of medical errors [1] [2] [3], to improve patient safety [4] [5] and to play a key role in encouraging a systems-based approach to identify causes of adverse events and potential solutions [6] [7]. While these conferences have traditionally been used in surgical specialties [5], they have been adopted widely by a range of specialties, including internal medicine [1] [8] [9], emergency medicine [10], rehabilitation medicine [11], psychiatry [12] and intensive care [4]. A number of medical oncology departments have implemented chemotherapy-specific M\&M conferences as part of quality improvement initiatives and have found them to be helpful in educating trainees and addressing the unique challenges of cancer care [13] [14]. However, despite the increasing complexity of oncology care [15] [16], the adoption of oncology-specific M\&M conferences in cancer centers is not routine [17]. Despite being commonly used in general obstetrics and gynecology, there is little published about M\&M conferences in the management of chemotherapy-related complications in gynecologic oncology [18] [19] [20]. Traditional chemotherapy as well as newer biologics and immunotherapies used in the treatment of gynecologic cancers have real and important toxicities that can impact patient quality of life and lead to life-threatening complications [21]. As a significant proportion of patients with gynecologic cancers are treated with palliative intent [22], it is essential that quality of life and treatment complications are monitored and used to drive chemotherapy-treatment in this setting. Herein we report the results of a chemotherapy-specific Gynecologic Oncology morbidity and mortality rounds and detail the reasons for hospital admission less than 30 days after chemotherapy treatment for patients with gynecologic cancers.

\section{Methods}

Institutional research ethics board approval was obtained prior to commencing this study. Between July 2014 and April 2016, all patients admitted to the gynecologic oncology service at the Massachusetts General Hospital were screened each month by the clinical fellows on service. Those patients who were admitted less than 30 days after most recent chemotherapy administration were then identified for inclusion in the quality improvement $M \& M$ conference whereas patients who had not received recent chemotherapy ( $<30$ days) were excluded. 
Information was gathered on each patient including age, diagnosis, cancer stage, chemotherapy treatment history, reason for admission and length of stay. Patients were then further classified as having a planned admission, defined as an admission scheduled as an outpatient for a specific clinical indication, or an unplanned admission, which was an admission through the emergency department or a direct admission from clinic due to an acute medical issue. Each month, a one-hour long structured morbidity and mortality conference was given by the clinical fellows that included a presentation of monthly admission statistics as well as a discussion of relevant clinical cases. These conferences were attended by all gynecologic oncology fellows, residents and medical students on the gynecologic oncology service as well as attending physicians. Clinical fellows reviewed each case to determine if the admission diagnosis was related to chemotherapy administration or instead related to underlying disease burden. Cases where there was uncertainty in the relation between diagnosis and chemotherapy administration were reviewed in detail at the conference to obtain a group consensus. If patients were chosen for further discussion, additional clinical history including details of the current history, investigations, past medical and surgical history and social history were gathered to allow for a complete analysis of their particular case. Each month the clinical fellow also picked a learning topic for review based on the clinical case list and a review of relevant literature was presented. After the formal presentation, a discussion of the admissions and relevant cases was undertaken by all learners and faculty present to identify areas for future quality improvement.

Data from these morbidity and mortality conferences was then analyzed to determine trends in chemotherapy admission within the study period. Descriptive statistics were used for baseline cohort characteristics, with continuous variables described as means \pm standard deviations and ranges and categorical variables described as numbers and percentages. Continuous variables were compared using Students T-tests and categorical variables with Mann Whitney U tests, with a two-sided $\mathrm{p}$-value of $<0.05$ considered significant.

\section{Results}

Data was available for the months between July 2014 and April 2016. In this time there were 585 admissions to the inpatient gynecologic oncology service less than 30 days from chemotherapy administration, 348 from July 2014 to June 2015 (2014-2015) and 237 from July 2015 to April 2016 (2015-2016). On average, there were 27 admissions per month; however the admission rate varied by month with the highest number of admissions in April 2015 at 37 and the lowest in January 2016 at 16. Despite this fluctuation, there was no clear pattern identified with respect to number of admissions and time of year. There were no in-hospital deaths of admitted patients during the study period. In 2014-2015, 73\% of all chemotherapy admission were associated with an underlying diagnosis of ovarian cancer, while $10 \%$ were related to uterine cancer, $7 \%$ to cervical cancer, $4 \%$ 
to vulvar cancer, $4 \%$ to GTN and $<1 \%$ to vaginal cancers. In $2015-2016$, the numbers were similar at $83 \%$ ovarian, $8 \%$ uterine, $7 \%$ cervical and $2 \%$ vulvar cancers, respectively. Many patients (43\%) had recurrent disease, while $14 \%$ had stage IV disease, $33 \%$ had stage III disease and the remainder had early stage disease (5\% stage II and 4\% stage I). The average length of stay for the entire cohort was 3.9 \pm 1.9 days. The average age of the entire cohort was $61.5 \pm 3.5$ years. The most common chemotherapy administered prior to admission was intravenous carboplatin and paclitaxel. Other chemotherapy regimens administered and the number of patients who received each regimen are listed in Table 1.

Overall, 310 (53\%) patients had planned admissions, and 275 (47\%) had unplanned admissions. The average age was similar for patients undergoing planned versus unplanned admissions at $61.2 \pm 5.1$ years and $61.8 \pm 1.2$ years respectively. Unplanned admissions tended to be significantly longer at an average of $5.6 \pm 0.9$ days versus $2.4 \pm 1.2$ days for planned admissions $(\mathrm{p}=0.0003$ ) For patients who had a planned admission in 2014-2015, inpatient chemotherapy desensitization was the most common reason for admission (103 patients, $53 \%)$. Intra-peritoneal chemotherapy administration with 24-hour paclitaxel infusion was the second most common reason for admission (34 patients, 17.5\%), followed by chemoradiation (26 patients, 13.4\%). Other inpatient chemotherapy protocols included EMA-CO (etoposide, methotrexate, actinomycin D, cyclophosphamide, vincristine) or BEP (bleomycin, etoposide, cisplatin) (20 patients, 10.3\%). Admission after neoadjuvant chemotherapy for surgical cytoreduction (11 patients, 5.6\%) was also included (Figure 1). Reasons for planned admissions were similar in 2015-2016.

Table 1. Chemotherapy regimens administered prior to admission $(\mathrm{n}=585)$.

\begin{tabular}{cc}
\hline Chemotherapy & Number of patients, $\mathbf{n}(\%)$ \\
\hline IV Carboplatin + IV Paclitaxel & $58(16.6 \%)$ \\
IV/IP Cisplatin + Paclitaxel & $52(14.9 \%)$ \\
Single-agent Carboplatin & $50(14.4 \%)$ \\
Single-agent Cisplatin & $28(8 \%)$ \\
Cisplatin + 5-fluorouracil & $19(5.5 \%)$ \\
Liposomal doxorubicin & $17(4.9 \%)$ \\
Carboplatin + liposomal doxorubicin & $15(4.3 \%)$ \\
Paclitaxel + bevacizumab & $15(4.3 \%)$ \\
EMA-CO & $13(3.7 \%)$ \\
Single-agent paclitaxel & $13(3.7 \%)$ \\
Gemcitabine & $9(2.6 \%)$ \\
Carboplatin + Gemcitabine & $6(1.7 \%)$ \\
Other, including trials & $53(15.2 \%)$ \\
\hline
\end{tabular}

${ }^{\star} \mathrm{IV}=$ intravenous, IP = intraperitoneal, EMA-CO = etoposide, methotrexate, actinomycin-D, cisplatin, vincristine. 
With respect to unplanned admissions in 2014-2015, the most common cause overall was nausea and vomiting or failure to thrive at home. Fifty-five patients were admitted with this diagnosis, representing $35.7 \%$ of the cohort. Other common causes were small bowel obstruction (29 patients, $18.8 \%$ ) and fever, infection or febrile neutropenia (26 patients, 16.9\%). Six patients (3.9\%) were admitted with a large bowel obstruction and 7 patients $(4.5 \%)$ with venous thromboembolic complications. Eight patients (5.2\%) were admitted with altered mental status due to dehydration and electrolyte abnormalities while three patients were admitted for renal failure (1.9\%). The remainder of the reasons for admission are shown in Figure 2. In 2015-2016, the causes of unplanned admission were similar with $22 \%$ of patients being admitted for nausea, vomiting and

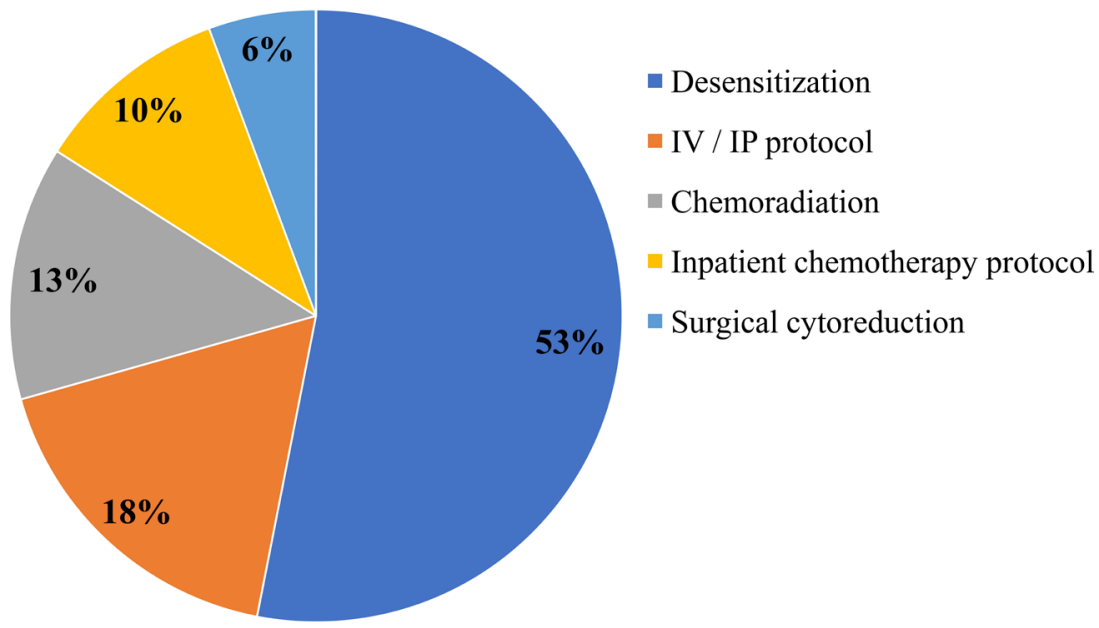

${ }^{\star} \mathrm{IV} / \mathrm{IP}=$ intravenous/intra-peritoneal.

Figure 1. Reasons for planned chemotherapy admission in 2014-2015 ( $\mathrm{n}=194)$.

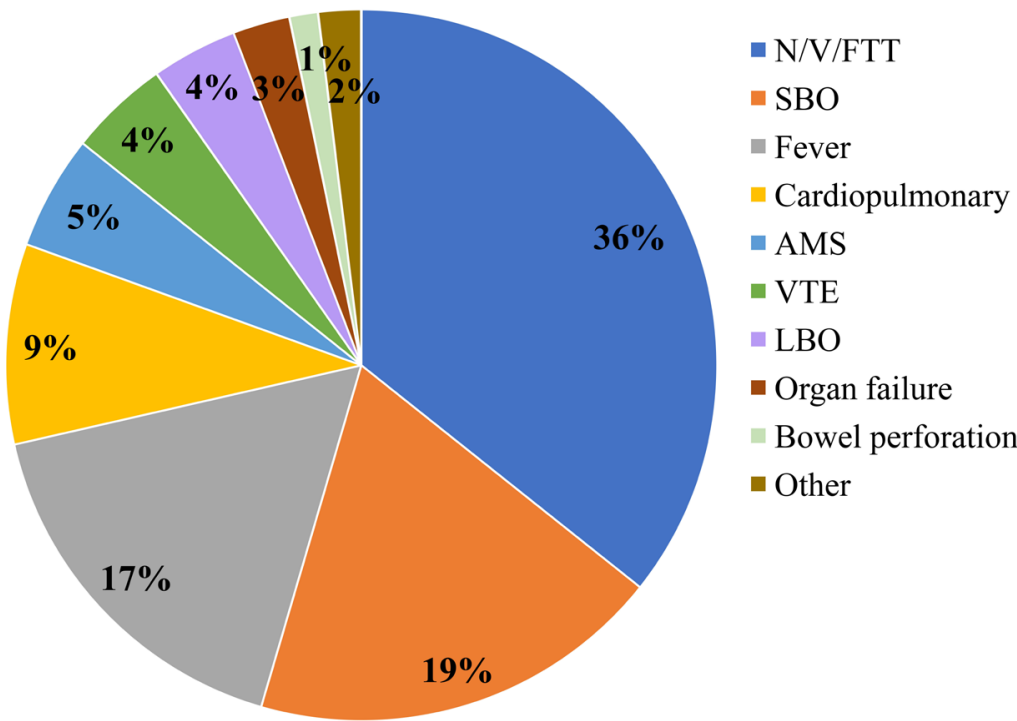

${ }^{*} \mathrm{~N} / \mathrm{V} / \mathrm{FTT}=$ nausea, vomiting, failure to thrive, $\mathrm{SBO}=$ small bowel obstruction, AMS $=$ altered mental status, VTE = venous thromboembolism, $\mathrm{LBO}=$ large bowel obstruction.

Figure 2. Reasons for unplanned chemotherapy admission in 2014-2015 ( $\mathrm{n}=154)$. 
failure to thrive at home, $21 \%$ for small bowel obstruction and $19 \%$ for fever, infection or febrile neutropenia. Procedural complications such as complications after line or gastrostomy/nephrostomy tube placement accounted for $5 \%$ of admissions in this time period. Overall, $43 \%$ percent of all unscheduled admissions were directly related to chemotherapy administration, and 57\% were unrelated to chemotherapy administration and occurred secondary to complications of disease burden. Seventy-four percent of patients having an unscheduled admission had received more than 1 previous line of chemotherapy, and $22 \%$ of patients were on clinical trial at the time of their admission.

Topics covered for case discussion included general principles of chemotherapy, management of hypersensitivity reactions, management of myelosuppression including neutropenic fever, chemotherapy related bowel perforations, management of nausea and vomiting, thromboembolism, chemotherapy in elderly populations and chemotherapy-associated liver, renal, cardiac and pulmonary toxicity.

\section{Discussion}

Morbidity and mortality (M\&M) rounds have been shown in multiple medical specialities to be an effective way to discuss complications associated with medical treatment [1] [2] [3], to identify systems-based solutions [6] [7] and to promote a culture of patient safety [4] [5]. Our prospective experience detailing results from a chemotherapy-specific gynecologic oncology M\&M conference identified a high rate of unplanned hospital admissions less than 30 days after chemotherapy admission, which was most pronounced in patients with advanced or recurrent disease. Furthermore, we found that this chemotherapy-specific conference was important in identifying trends associated with chemotherapy admissions and promoting resident and fellow education about both treatment paradigms and common complications.

Of the 585 admissions in our study, 53\% were planned admissions with inpatient chemotherapy desensitization representing the most common reason for planned admission in this cohort. The high rate of desensitization in our cohort is consistent with known rates of hypersensitivity after treatment with platinum $(10 \%-27 \%)$ [23] and taxane (5\% - 10\%) [24] chemotherapy, most notably as our cohort included a large number of patients with recurrent disease undergoing platinum re-challenge (43\%) [25]. This finding was relevant as a particularly important aspect of our M\&M meeting was the educational session structured around common themes of our monthly admissions. It was during this time that we were able to educate trainees about important chemotherapy issues, such as hypersensitivity reactions and desensitization protocols, which is paramount as if untreated, these reactions can progress to fatal anaphylactic shock [23]. In addition to encouraging relevant case-based education, we found that reviewing our planned admissions was also helpful in identifying trends in resource utilization. For instance, we found a high rate of admission (17.5\%) re- 
lated to intra-peritoneal chemotherapy (IP) with 24-hour paclitaxel infusion [26], which resulted in approximately 54 hospital admissions over 22 months. Effective outpatient IP chemotherapy regimens are available which have been shown to result in less toxicity, improved cycle completion rates and fewer hospital admissions [27] [28]. While the additional cost and strain of these admissions on the inpatient care providers may be warranted, we believe that through M\&M rounds, opportunities to streamline care such as this can be more easily addressed.

Overall, $47 \%$ of patients were admitted to our service due to unplanned acute complications. Less than half of these (43\%) admissions were found to be directly associated with chemotherapy, while the majority were associated with complications of disease burden such as large and small bowel obstructions. We believe that systematically reviewing these unplanned admissions is essential to both improving the management of common chemotherapy-associated complications and the care of our patients with advanced disease. Nausea and vomiting accounted for $28.9 \%$ of all unplanned admissions in our cohort, a complication which significantly reduces patient quality of life during treatment [29]. While the risk of chemotherapy-associated nausea and vomiting is related to a number of underlying factors including the type of chemotherapy [30], the use of combination prophylaxis with steroids, 5-HT3 receptor antagonists, NK-receptor antagonists and anti-psychotics such as olanzapine during the entire risk period can significantly reduce the occurrence of this complication [29] [31]. In response to the high rate of these admissions identified, we were able to standardize our outpatient anti-emetic treatment regimen to reduce the severity of symptoms in our patients moving forward. We believe that opportunities to improve patient care are at the heart of an M\&M program and are the most important outcomes that all patient care teams should work towards.

Additionally, we found that our M\&M rounds were useful to identify common treatment patterns in our patient population and to encourage providers to discuss treatment dilemmas in a confidential forum of peers. Up to $74 \%$ of our patients with an unscheduled admission had received more than 1 previous line of chemotherapy, and $22 \%$ were on a clinical trial at the time of admission. The majority of our patients with unscheduled admissions had therefore recurred, had advanced intra-peritoneal disease and had been heavily pretreated with multiple prior lines of chemotherapy at their time of presentation. Chemotherapy in this setting is intended to alleviate symptoms and improve quality of life in patients with incurable disease [32] however it has also been associated with significant toxicity, higher rate of hospital admission and an overall worse of quality of life [32]-[38]. Determining when treatment is no longer helpful and is potentially harmful can be challenging for providers, especially in those patients who desire continued aggressive treatment despite a low likelihood of clinical benefit. We believe that clearly addressing the toxicity associated with treatment in patients with recurrent disease can be helpful in promoting thoughtful, pa- 
tient-centered care and encouraging the early and consistent use of palliative care for symptom management [39] [40]. Furthermore, we believe that due to these M\&M conferences, our fellows became more adept at managing these complexities of chemotherapy care and significantly improved their comfort with goals of care conversations and treatment-related decision making. We have seen them become empowered as members of the health-care team to ask important questions regarding when to stop treatment in the case of futility and excessive toxicity.

In conclusion, we found that chemotherapy-specific morbidity and mortality conferences are useful, not only for identifying and discussing complications, but also for analyzing practice patterns associated with chemotherapy administration and determining areas for potential improvement. They also have been vital in improving trainee education and empowering them to collaborate with patients and staff oncology providers to make important chemotherapy-related healthcare decisions. As chemotherapy can be associated with significant morbidity in patients with gynecologic cancer, we believe that having forum to specifically address these complications is important. As this was a pilot project, our study does have the limitations of a short time course and limited data regarding provider satisfaction; however we hope to continue with these rounds to gather more detailed and useful information.

$M \& M$ rounds are used widely across surgical specialties, and we believe that the lack of a similar formal M\&M conference for chemotherapy represents the loss of an opportunity to review complications of an intervention that is similarly complex and potentially dangerous. It is for this reason that we believe that the routine implementation of chemotherapy-specific morbidity and mortality rounds in gynecologic oncology is essential to improve patient-centered care and reduce treatment associated morbidity in our patients.

\section{Acknowledgements}

Dr. Lauren Philp prepared the manuscript and was involved in manuscript editing and data analysis. Dr. Tracilyn Hall was involved in data collection, data analysis and manuscript editing. Dr. Elisabeth Diver was involved in data collection, data analysis and manuscript editing. Dr. Annekathryn Goodman is the senior author who developed the idea for the study, oversaw data collection and data analysis and performed manuscript editing.

\section{Conflicts of Interest}

The authors declare no conflicts of interest regarding the publication of this paper.

\section{References}

[1] Orlander, J.D., Barber, T.W. and Fincke, B.G. (2002) The Morbidity and Mortality Conference: The Delicate Nature of Learning from Error. Academic Medicine, 77, 
1001-1006. https://doi.org/10.1097/00001888-200210000-00011

[2] Gore, D.C. (2006) National Survey of Surgical Morbidity and Mortality Conferences. The American Journal of Surgery, 191, 708-714.

https://doi.org/10.1016/j.amjsurg.2006.01.029

[3] Volpp, K.G.M. and Grande, D. (2003) Residents' Suggestions for Reducing Errors in Teaching Hospitals. The New England Journal of Medicine, 348, 851-855. https://doi.org/10.1056/NEJMsb021667

[4] Frey, B., Doell, C., Klauwer, D., et al. (2016) The Morbidity and Mortality Conference in Pediatric Intensive Care as a Means for Improving Patient Safety. Pediatric Critical Care Medicine, 17, 67-72. https://doi.org/10.1097/PCC.0000000000000550

[5] Bal, G., Sellier, E., Tchouda, S.D. and François, P. (2014) Improving Quality of Care and Patient Safety through Morbidity and Mortality Conferences. Journal for Healthcare Quality, 36, 29-36. https://doi.org/10.1111/j.1945-1474.2011.00203.x

[6] Szostek, J., Wieland, M., Loertscher, L., et al. (2010) A Systems Approach to Morbidity and Mortality Conference. The American Journal of Medicine, 123, 663-668. https://doi.org/10.1016/j.amjmed.2010.03.010

[7] Cross, S.R.H. (2018) The Systems Approach at the Sharp End. Future Healthcare Journal, 5, 176-180. https://doi.org/10.7861/futurehosp.5-3-176

[8] Tapper, E.B. and Leffler, D.A. (2016) The Morbidity and Mortality Conference in Gastroenterology and Hepatology: An Important Cornerstone of Patient Safety and Optimal Care. Gastroenterology, 150, 19-23. https://doi.org/10.1053/j.gastro.2015.11.027

[9] McNamara, D.A., Hall, H.M. and Hardin, E.A. (2019) Rethinking the Modern Cardiology Morbidity and Mortality Conference: Harmonizing Quality Improvement and Education. Journal of the American College of Cardiology, 73, 868-872. https://doi.org/10.1016/j.jacc.2019.01.007

[10] Seigel, T.A., McGillicuddy, D.C., Barkin, A.Z. and Rosen, C.L. (2010) Morbidity and Mortality Conference in Emergency Medicine. The Journal of Emergency Medicine, 38, 507-511. https://doi.org/10.1016/j.jemermed.2008.09.018

[11] Esselman, P.C. and Dillman-Long, J. (2002) Morbidity and Management Conference: An Approach to Quality Improvement in Brain Injury Rehabilitation. Journal of Head Trauma Rehabilitation, 17, 257-262. https://doi.org/10.1097/00001199-200206000-00007

[12] Hollanda, J. (2007) A Role for Morbidity and Mortality Conferences in Psychiatry. Australasian Psychiatry, 15, 338-342. https://doi.org/10.1080/10398560701351803

[13] Goodman, L., Moeller, M., Guthrie, A., Earl, M., Pennel, N. and Gilligan, T. (2017) The Morbidity and Mortality Conference (MMC) in Oncology: A Patient Safety and root Cause Analysis (RCA)-Driven Approach at the Cleveland Clinic Taussig Cancer Institute (TCI). Journal of Clinical Oncology, 32, 191-191. https://doi.org/10.1200/jco.2014.32.30_suppl.191

[14] Stover, D.G. and Zerillo, J.A. (2015) Morbidity and Mortality Revisited: Applying a New Quality Improvement Paradigm in Oncology. Journal of Oncology Practice, 11, e428-e433. https://doi.org/10.1200/JOP.2014.003566

[15] Shulman, L.N., Jacobs, L.A., Greenfield, S., et al. (2009) Cancer Care and Cancer Survivorship Care in the United States: Will We Be Able to Care for These Patients in the Future? Journal of Oncology Practice, 5, 119-123.

https://doi.org/10.1200/JOP.0932001

[16] (2106) Oncology AS of C. The State of Cancer Care in America, 2016: A Report by 
the American Society of Clinical Oncology. Journal of Oncology Practice, 12, 339-383. https://doi.org/10.1200/JOP.2015.010462

[17] Belkacemi, Y., Colson-Durand, L., Fayolle-Campana, M., et al. (2019) A Wake-Up Call for Routine Morbidity and Mortality Review Meeting Procedures as Part of a Quality Governance Programs in Radiation Therapy Departments: Results of the PROUST Survey. Practical Radiation Oncology, 9, 108-114. https://doi.org/10.1016/j.prro.2018.09.004

[18] Jackson, J. and De Cesare, J. (2015) Multidisciplinary OBGYN Morbidity and Mortality Conference. Archives of Gynecology and Obstetrics, 292, 7-11. https://doi.org/10.1007/s00404-015-3710-5

[19] Po, L., Durand, N. and Page, A. (2019) Implementation of Structured Morbidity and Mortality Rounds in Obstetrics and Gynecology. Journal of Obstetrics and Gynaecology Canada, 41, 730. https://doi.org/10.1016/j.jogc.2019.02.225

[20] Bevis, K.S., Straughn, J.M., Kendrick, J.E., Walsh-Covarrubias, J. and Kilgore, L.C. (2011) Morbidity and Mortality Conference in Obstetrics and Gynecology: A Tool for Addressing the 6 Core Competencies. The Journal of Graduate Medical Education, 3, 100-103. https://doi.org/10.4300/JGME-D-10-00093.1

[21] Rojas, C. and Casablanca, Y. (2019) Chemotherapy, Biologic, and Immunotherapy Breakthroughs in Cancer Care. Obstetrics and Gynecology Clinics of North America, 46, 137-154. https://doi.org/10.1016/j.ogc.2018.09.009

[22] Penson, R.T., Wenzel, L., Vergote, I. and Cella, D. (2006) Quality of Life Considerations in Gynecologic Cancer. International Journal of Gynecology \& Obstetrics, 95, s247-s257. https://doi.org/10.1016/S0020-7292(06)60040-4

[23] Shepherd, G.M. (2003) Hypersensitivity Reactions to Chemotherapeutic Drugs. Clinical Reviews in Allergy \& Immunology, 24, 253-262. https://doi.org/10.1385/CRIAI:24:3:253

[24] Picard, M. (2017) Management of Hypersensitivity Reactions to Taxanes. Immunology and Allergy Clinics of North America, 37, 679-693. https://doi.org/10.1016/j.iac.2017.07.004

[25] Boulanger, J., Boursiquot, J.N., Cournoyer, G., et al. (2014) Management of Hypersensitivity to Platinum- and Taxane-Based Chemotherapy: CEPO Review and Clinical Recommendations. Current Oncology, 21, 630-641. https://doi.org/10.3747/co.21.1966

[26] Armstrong, D.K., Bundy, B., Wenzel, L., et al. (2006) Intraperitoneal Cisplatin and paclitaxel in Ovarian Cancer. The New England Journal of Medicine, 354, 34-43. https://doi.org/10.1056/NEJMoa052985

[27] Berry, E., Matthews, K., Singh, D., Buttin, B., Lurain, J. and Alvarez, R. (2009) An Outpatient Intraperitoneal Chemotherapy Regimen for Advanced ovarian Cancer. Gynecologic Oncology, 113, 63-67. https://doi.org/10.1016/j.ygyno.2008.12.035

[28] Seamon, L., Carlson, M., Richardson, D., et al. (2009) Outpatient Platinum-Taxane Intra-Peritoneal Chemotherapy Regiment for Ovarian Cancer. International Journal of Gynecologic Cancer, 19, 1195-1198. https://doi.org/10.1111/IGC.0b013e3181b33d5b

[29] Navari, R.M. and Aapro, M. (2016) Antiemetic Prophylaxis for Chemotherapy-Induced Nausea and Vomiting. The New England Journal of Medicine, 374, 1356-1367. https://doi.org/10.1056/NEJMra1515442

[30] Mizuno, M., Hiura, M., Kikkawa, F., et al. (2016) A Prospective Observational Study on Chemotherapy-Induced Nausea and Vomiting (CINV) in Patients with Gyne- 
cologic Cancer by the CINV Study Group of Japan. Gynecologic Oncology, 140, 559-564. https://doi.org/10.1016/j.ygyno.2015.12.029

[31] Shimokawa, M., Hayashi, T., Kogawa, T., et al. (2019) Evaluation of Combination Anti-Emetic Therapy on CINV in Patients with Gynecologic Cancer Receiving TC Chemotherapy. Anticancer Research, 39, 225-230. https://doi.org/10.21873/anticanres.13101

[32] Jang, T.K., Kim, D.Y., Lee, S.W., et al. (2018) Trends in Treatment during the Last Stages of Life in End-Stage Gynecologic Cancer Patients Who Received Active Palliative Chemotherapy: A Comparative Analysis of 10-Year Data in a Single Institution. BMC Palliative Care, 17, 1-8. https://doi.org/10.1186/s12904-018-0348-7

[33] Sompratthana, T., Phoolcharoen, N., Schmeler, K. and Lertkhachonsuk, R. (2019) End-of-Life Symptoms and Interventions among Women with Gynecologic Cancers in a Tertiary-Care Hospital in Thailand. International Journal of Gynecologic Cancer, 29, No. 5. https://doi.org/10.1136/ijgc-2019-000338

[34] Näppä, U., Lindqvist, O., Rasmussen, B.H. and Axelsson, B. (2011) Palliative Chemotherapy during the Last Month of Life. Annals of Oncology, 22, 2375-2380. https://doi.org/10.1093/annonc/mdq778

[35] Prigerson, H.G., Bao, Y., Shah, M.A., et al. (2015) Chemotherapy Use, Performance Status, and Quality of Life at the End of Life. JAMA Oncology, 1, 778-784. https://doi.org/10.1001/jamaoncol.2015.2378

[36] Roncolato, F.T., Joly, F., O’Connell, R., et al. (2017) Reducing Uncertainty: Predictors of Stopping Chemotherapy Early and Shortened Survival Time in Platinum Resistant/Refractory Ovarian Cancer-The GCIG Symptom Benefit Study. The Oncologist, 22, 1117-1124. https://doi.org/10.1634/theoncologist.2017-0047

[37] Friedlander, M.L., Stockler, M., O’Connell, R., et al. (2014) Symptom Burden and Outcomes of Patients with Platinum Resistant/Refractory Recurrent Ovarian Cancer: A Reality Check: Results of Stage 1 of the Gynecologic Cancer Intergroup Symptom Benefit Study. International Journal of Gynecologic Cancer, 24, 857-864. https://doi.org/10.1097/IGC.0000000000000147

[38] Rha, S.Y. and Lee, J. (2017) Symptom Clusters during Palliative Chemotherapy and Their Influence on Functioning and Quality of Life. Supportive Care in Cancer, 25, 1519-1527. https://doi.org/10.1007/s00520-016-3545-Z

[39] Rocque, G.B. and Cleary, J.F. (2013) Palliative Care Reduces Morbidity and Mortality in Cancer. Nature Reviews Clinical Oncology, 10, 80-89. https://doi.org/10.1038/nrclinonc.2012.211

[40] Roy, M. and Ramchandran, K. (2020) Symptom Relief and Palliative Care in Gynecologic Oncology. Current Opinion in Obstetrics and Gynecology, 32, 65-75. https://doi.org/10.1097/GCO.0000000000000601 